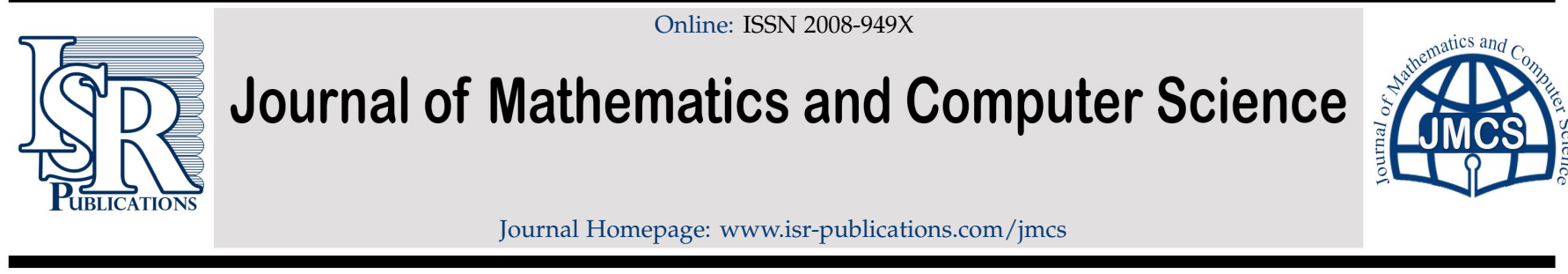

\title{
Generalized fractional delay functional equations with Riemann-Stieltjes and infinite point nonlocal conditions
}

\author{
M. I. Youssef \\ Department of Mathematics, College of Science, Jouf University, P. O. Box 2014, Sakaka, Saudi Arabia. \\ Department of Mathematics, Faculty of Education, Alexandria University, Alexandria, Egypt.
}

\begin{abstract}
In the current work, we investigate the solvability of a general class of fractional delay functional equations subject to an infinite point non-classical condition, and the Riemann-Stieltjes integral condition as well. First, the existence of solutions is investigated. Second, the continuous dependence of solution is studied in three different cases. Third, illustrative examples are given to support our results. Our work extends some developments published recently in that field.
\end{abstract}

Keywords: Fractional integro-differential equation, existence of solutions, infinite point non-classical condition, Riemann-Stieltjes nonlocal condition, delay function.

2020 MSC: 34A12, 45D05, 34K37.

(C)2022 All rights reserved.

\section{Introduction}

Studying functional integro-differential equations is of significance, since integro-differential equations can describe successfully many applications in biological, chemical, and physical sciences. For example, the mathematical models in biological populations depend on Volterra integro-differential equations with delay, the behavior of the nuclear reactor in a continuous medium can be described by a system of integrodifferential equations, and many other problems in economics, mechanics, visco-elasticity as well. Also, these types of equations occur when we convert the Cauchy and boundary value problems to integral equations, (see $[3,5,6,15]$ and the cited references therein for more explanations).

The nonlocal conditions give more initial measurements which are more precise than the local condition. So, these conditions allow us to get more accurate model of the phenomena being studied and therefore, better results can be derived. Recently, the study of existence of solutions for initial value problems subject to non-classical conditions such as the Riemann-Stieltjes and the infinite point nonlocal conditions becomes an issue of great importance, see $[14,18]$. As a continuation in that progress, ElSayed et al. studied in [10] the evolution of a physical model described by a nonlocal integro-differential equation on the form

$$
\frac{d z}{d t}=g\left(t, z(t), \int_{0}^{t} h(\tau, z(\tau)) d \tau\right), \quad \text { a.e. } t \in(0,1)
$$

Email address: miyoussef283@gmail . com (M. I. Youssef)

doi: $10.22436 /$ jmcs.024.01.04

Received: 2020-07-21 Revised: 2020-09-22 Accepted: 2020-11-20 
subject to the condition

$$
\sum_{k=1}^{m} b_{k} z\left(\tau_{k}\right)=z_{0}, \quad b_{k} \geqslant 0, \tau_{k} \in(0,1)
$$

The authors proved the existence of continuous solutions to the model characterized by Eqs. (1.1)-(1.2) provided some sufficient conditions are verified. They studied the data dependence of solutions and, moreover, investigated Eq. (1.1) subject to the Riemann-Stieltjes integral condition

$$
\int_{0}^{1} z(\tau) \operatorname{dh}(\tau)=z_{0}
$$

and the infinite-point condition

$$
\sum_{k=1}^{\infty} b_{k} z\left(\tau_{k}\right)=z_{0}
$$

to demonstrate the applicability of their model. In this article, we study a more general fractional evolution model of Eqs. (1.1)-(1.2) which was not discussed in [10]. This fractional model has many real world applications in environmental science, electrochemistry, viscoelasticity, dynamical systems, biomedicine, control theory and signal processing, see $[4,8,16,17]$. Consequently, we are concerned with the existence of solutions for a generalized fractional nonlinear physical delayed model described by

$$
\left({ }^{C} D_{0^{+}}^{\beta, \rho}-\Lambda\right) z(t)=\sum_{i=1}^{N} g_{i}\left(t, \mathcal{L}(z)(t), \int_{0}^{\Phi(t)} h(\tau, z(\tau)) d \tau\right), \quad \text { a.e. } t \in(0, T],
$$

subject to the following three cases of nonlocal conditions

$$
\begin{aligned}
& \sum_{k=1}^{m} b_{k} z\left(\tau_{k}\right)=z_{0}, \quad b_{k}>0, \tau_{k} \in(0, T], \\
& \sum_{k=1}^{\infty} b_{k} z\left(\tau_{k}\right)=z_{0}, \quad b_{k}>0, \tau_{k} \in(0, T], \\
& \int_{0}^{T} z(\tau) d \hbar(\tau)=z_{0},
\end{aligned}
$$

where ${ }^{C} D_{0^{+}}^{\beta, \rho}$ denotes the left-sided Caputo-Katugampola fractional derivative [2, 13]. The parameters $\beta \in(0,1)$, and $\rho \in \mathbb{R}_{>0}:=\{x \in \mathbb{R}: x>0\}$ such that $\rho \beta>1$. The function $\Phi(t)$ is called the vanishing lag function and defined by $\Phi(\mathrm{t}):=(\mathrm{t}-\varphi(\mathrm{t}))$, with a fading lag $\varphi(\mathrm{t})$, where $\varphi(0)=0$, and $0<\varphi(\mathrm{t})<\mathrm{t}, \forall \mathrm{t} \in$ $J:=[0, T] \backslash\{0\},[7]$. The operators $\Lambda$ and $\mathcal{L}$ are linear closed self-operators on $C(J, \mathbb{R})$. The functions $g_{i}$, $1 \leqslant i \leqslant N<\infty, \Phi$ and $h$ are assumed to be measurable with respect to $t$ on J preserving some properties which will be stated in Section 2. The next sections of this work are organized as follows. Sections 2-6 are devoted to the main results regarding the existence, and continuous dependence of solution. We give two illustrative examples in Section 7 to support the importance of the current work. In Section 8, we present a conclusion and some suggested future work.

\section{Existence results}

Applying the definition of generalized fractional integral due to the Katugampola in [12] to both sides of Eq. (1.3), then utilizing theorem 6 in [16] formally yields

$$
\begin{aligned}
z(t)= & z(0)+\frac{\rho^{-\beta}}{\Gamma(\beta)} \int_{0}^{t}\left(t^{\rho}-\zeta^{\rho}\right)^{\beta-1} \Lambda(z)(\zeta) d \zeta^{\rho} \\
& +\frac{\rho^{-\beta}}{\Gamma(\beta)} \sum_{i=1}^{N} \int_{0}^{t}\left(t^{\rho}-\zeta^{\rho}\right)^{\beta-1} g_{i}\left(\zeta, \mathcal{L}(z)(\zeta), \int_{0}^{\Phi(\zeta)} h(s, z(s)) d s\right) d \zeta^{\rho} .
\end{aligned}
$$


Using condition (1.4), and let $A:=\sum_{k=1}^{m} b_{k}$, where $A \neq 0$, it is easy to obtain the following integral representation for $z(t)$,

$$
\begin{aligned}
z(t)= & A^{-1}\left[z_{0}-\frac{\rho^{-\beta}}{\Gamma(\beta)} \sum_{k=1}^{m} b_{k} \int_{0}^{\tau_{k}}\left(\tau_{k}^{\rho}-\zeta^{\rho}\right)^{\beta-1} \Lambda(z)(\zeta) d \zeta^{\rho}\right]+\frac{\rho^{-\beta}}{\Gamma(\beta)} \int_{0}^{t}\left(t^{\rho}-\zeta^{\rho}\right)^{\beta-1} \Lambda(z)(\zeta) d \zeta^{\rho} \\
& -\frac{A^{-1} \rho^{-\beta}}{\Gamma(\beta)} \sum_{k=1}^{m} \sum_{i=1}^{N} b_{k} \int_{0}^{\tau_{k}}\left(\tau_{k}^{\rho}-\zeta^{\rho}\right)^{\beta-1} g_{i}\left(\zeta, \mathcal{L}(z)(\zeta), \int_{0}^{\Phi(\zeta)} h(s, z(s)) d s\right) d \zeta^{\rho} \\
& +\frac{\rho^{-\beta}}{\Gamma(\beta)} \sum_{i=1}^{N} \int_{0}^{t}\left(t^{\rho}-\zeta^{\rho}\right)^{\beta-1} g_{i}\left(\zeta, \mathcal{L}(z)(\zeta), \int_{0}^{\Phi(\zeta)} h(s, z(s)) d s\right) d \zeta^{\rho}
\end{aligned}
$$

Definition 2.1 ([11]). A solution for the problem (1.3) under the nonlocal condition (1.4) is called a mild solution if it satisfies the integral equation (2.1).

In what follows, let the following conditions be satisfied.

(V1) The mappings $g_{i}, 1 \leqslant i \leqslant N<\infty$, are non-self mappings from $J \times \mathbb{R}^{2}$ into $\mathbb{R}$ and have the following attributes

(a) $\forall i \in\{1,2, \cdots, N\}$ the function $g_{i}(t, \cdot, \cdot)$ is continuous on $\mathbb{R}^{2}$ for almost all $t \in J$;

(b) $\exists$ a function $b_{i} \in \mathrm{L}^{1}([0, \mathrm{~T}]), i \in\{1,2, \cdots, \mathrm{N}\}$ and two constants $v_{i} \in \mathbb{R}_{>0}:=\{x \in \mathbb{R}: x>0\}$, $i \in\{1,2\}$, such that for almost all $(t, y, z) \in J \times \mathbb{R}^{2}$ we have

$$
\left|g_{i}(t, y, z)\right| \leqslant b_{i}(t)+v_{1}|y|+v_{1}|z|, \quad \sup _{t \in J} I_{0^{+}}^{\beta, \rho} b_{i}(t) \leqslant v_{2}, \quad \forall i \in\{1,2, \cdots, N\} .
$$

(V2) The mapping $h$ is defined on $J \times \mathbb{R}$ with values in $\mathbb{R}_{>0}$ such that:

(a) $\forall t \in J$, the function $h(t, \cdot)$ is continuous on $\mathbb{R}$;

(b) $\forall(t, z) \in J \times \mathbb{R}, \exists \vartheta_{i}>0, i \in\{1,2\}$ such that $|h(t, z)| \leqslant \vartheta_{1}+\vartheta_{2}|z|$.

(V3) $\rho^{\beta} \Gamma(1+\beta)>2 \eta T^{\rho \beta}$, where $\eta:=\gamma\left(1+v_{1} N\right)+v_{1} \vartheta_{2} N T>0$ and $\gamma>0$.

(V4) The vanishing delay function $\Phi$ is a continuous self-mapping on J.

Theorem 2.2. Suppose the hypotheses V1-V4 are fulfilled. Then the nonlocal problem (1.3)-(1.4) has at least one continuous mild solution.

Proof. Define an operator W corresponding to the integral equation (2.1) by

$$
\begin{aligned}
W(z)(t)= & A^{-1} z_{0}-\frac{A^{-1} \rho^{-\beta}}{\Gamma(\beta)} \sum_{k=1}^{m} b_{k} \int_{0}^{\tau_{k}}\left(\tau_{k}^{\rho}-\zeta^{\rho}\right)^{\beta-1} \Lambda(z)(\zeta) d \zeta^{\rho}+\frac{\rho^{-\beta}}{\Gamma(\beta)} \int_{0}^{t}\left(t^{\rho}-\zeta^{\rho}\right)^{\beta-1} \Lambda(z)(\zeta) d \zeta^{\rho} \\
& -\frac{A^{-1} \rho^{-\beta}}{\Gamma(\beta)} \sum_{k=1}^{m} \sum_{i=1}^{N} b_{k} \int_{0}^{\tau_{k}}\left(\tau_{k}^{\rho}-\zeta^{\rho}\right)^{\beta-1} g_{i}\left(\zeta, \mathcal{L}(z)(\zeta), \int_{0}^{\Phi(\zeta)} h(s, z(s)) d s\right) d \zeta^{\rho} \\
& +\frac{\rho^{-\beta}}{\Gamma(\beta)} \sum_{i=1}^{N} \int_{0}^{t}\left(t^{\rho}-\zeta^{\rho}\right)^{\beta-1} g_{i}\left(\zeta, \mathcal{L}(z)(\zeta), \int_{0}^{\Phi(\zeta)} h(s, z(s)) d s\right) d \zeta^{\rho} .
\end{aligned}
$$

So, to prove the existence of at least one solution to the integral equation (2.1), it is sufficient to show the functional equation (2.2) has at least one fixed point

$$
z=W(z)
$$

Consider the set $S_{r}:=\left\{z \in C(J, \mathbb{R}):\|z\|_{\infty}=\sup _{t \in J}\{|z(t)|\} \leqslant r\right\}$, where the radius $r$ is given by $r:=$ $\frac{\left(A^{-1}\left|z_{0}\right|+2 v_{2} N\right) \Gamma(1+\beta)+2 v_{1} \vartheta_{1} N \rho^{-\beta} T^{(1+\rho \beta)}}{\Gamma(1+\beta)-2 \eta \rho^{-\beta} T^{\rho \beta}}$. 
Step $1 \mathrm{in}$ the proof is to show the operator $W$ is a self-operator of the set $S_{\mathrm{r}}$ as follows.

$$
\begin{aligned}
|W(z)(t)| \leqslant & A^{-1}\left|z_{0}\right|+\frac{A^{-1} \rho^{-\beta}}{\Gamma(\beta)} \sum_{k=1}^{m} b_{k} \int_{0}^{\tau_{k}}\left(\tau_{k}^{\rho}-\zeta^{\rho}\right)^{\beta-1}\|\Lambda(z)\|_{\infty} d \zeta^{\rho}+\frac{\rho^{-\beta}}{\Gamma(\beta)} \int_{0}^{t}\left(t^{\rho}-\zeta^{\rho}\right)^{\beta-1}\|\Lambda(z)\|_{\infty} d \zeta^{\rho} \\
& +\frac{A^{-1} \rho^{-\beta}}{\Gamma(\beta)} \sum_{k=1}^{m} \sum_{i=1}^{N} b_{k} \int_{0}^{\tau_{k}}\left(\tau_{k}^{\rho}-\zeta^{\rho}\right)^{\beta-1}\left|g_{i}\left(\zeta, \mathcal{L}(z)(\zeta), \int_{0}^{\Phi(\zeta)} h(s, z(s)) d s\right)\right| d \zeta^{\rho} \\
& +\frac{\rho^{-\beta}}{\Gamma(\beta)} \sum_{i=1}^{N} \int_{0}^{t}\left(t^{\rho}-\zeta^{\rho}\right)^{\beta-1}\left|g_{i}\left(\zeta, \mathcal{L}(z)(\zeta), \int_{0}^{\Phi(\zeta)} h(s, z(s)) d s\right)\right| d \zeta^{\rho} .
\end{aligned}
$$

The operator $\Lambda$ is a linear closed self-operator on the Banach space $\left(C(J, \mathbb{R}),\|\cdot\|_{\infty}\right)$. So, it is bounded from applying the closed graph theorem and hence $\|\Lambda(z)\|_{\infty} \leqslant \gamma_{1}\|z\|_{\infty}, \gamma_{1} \geqslant 0$. Also, $\|\mathcal{L}(z)\|_{\infty} \leqslant \gamma_{2}\|z\|_{\infty}, \gamma_{2} \geqslant 0$ from applying the closed graph theorem as well, see $[9,20]$. Now using conditions $V 1 b$ and $\gamma=\max \left\{\gamma_{i}\right.$ : $i=1,2\}$ implies

$$
\begin{aligned}
|W(z)(t)| \leqslant & A^{-1}\left|z_{0}\right|+\frac{A^{-1} \rho^{-\beta}}{\Gamma(\beta)} \sum_{k=1}^{m} b_{k} \int_{0}^{\tau_{k}}\left(\tau_{k}^{\rho}-\zeta^{\rho}\right)^{\beta-1} \gamma\|z\|_{\infty} d \zeta^{\rho}+\frac{\rho^{-\beta}}{\Gamma(\beta)} \int_{0}^{t}\left(t^{\rho}-\zeta^{\rho}\right)^{\beta-1} \gamma\|z\|_{\infty} d \zeta^{\rho} \\
& +\frac{A^{-1} \rho^{-\beta}}{\Gamma(\beta)} \sum_{k=1}^{m} \sum_{i=1}^{N} b_{k} \int_{0}^{\tau_{k}}\left(\tau_{k}^{\rho}-\zeta^{\rho}\right)^{\beta-1}\left[b_{i}(\zeta)+v_{1} \gamma\|z\|_{\infty}+v_{1} \int_{0}^{\Phi(\zeta)}|h(s, z(s))| d s\right] d \zeta^{\rho} \\
& +\frac{\rho^{-\beta}}{\Gamma(\beta)} \sum_{i=1}^{N} \int_{0}^{t}\left(t^{\rho}-\zeta^{\rho}\right)^{\beta-1}\left[b_{i}(\zeta)+v_{1} \gamma\|z\|_{\infty}+v_{1} \int_{0}^{\Phi(\zeta)}|h(s, z(s))| d s\right] d \zeta^{\rho} .
\end{aligned}
$$

Applying condition $\mathrm{V} 2 \mathrm{~b}$ and the properties of the lag function yields

$$
\begin{aligned}
|W(z)(t)| \leqslant & A^{-1}\left|z_{0}\right|+\frac{A^{-1} \gamma r \rho^{-\beta}}{\Gamma(\beta)} \sum_{k=1}^{m} b_{k} \int_{0}^{\tau_{k}}\left(\tau_{k}^{\rho}-\zeta^{\rho}\right)^{\beta-1} d \zeta^{\rho}+\frac{\gamma r \rho^{-\beta}}{\Gamma(\beta)} \int_{0}^{t}\left(t^{\rho}-\zeta^{\rho}\right)^{\beta-1} d \zeta^{\rho} \\
& +\frac{A^{-1} \rho^{-\beta}}{\Gamma(\beta)} \sum_{k=1}^{m} \sum_{i=1}^{N} b_{k} \int_{0}^{\tau_{k}}\left(\tau_{k}^{\rho}-\zeta^{\rho}\right)^{\beta-1}\left[b_{i}(\zeta)+v_{1} \gamma r+v_{1}\left(\vartheta_{1}+\vartheta_{2} r\right)(\Phi(\zeta)-\Phi(0))\right] d \zeta^{\rho} \\
& +\frac{\rho^{-\beta}}{\Gamma(\beta)} \sum_{i=1}^{N} \int_{0}^{t}\left(t^{\rho}-\zeta^{\rho}\right)^{\beta-1}\left[b_{i}(\zeta)+v_{1} \gamma r+v_{1}\left(\vartheta_{1}+\vartheta_{2} r\right)(\Phi(\zeta)-\Phi(0))\right] d \zeta^{\rho} .
\end{aligned}
$$

After calculating and simplifying the above integrals we obtain

$$
|W(z)(t)| \leqslant A^{-1}\left|z_{0}\right|+2 v_{2} N+\frac{2 \rho^{-\beta} T^{\rho \beta}}{\Gamma(1+\beta)}\left[v_{1} \vartheta_{1} N T+\eta r\right] .
$$

Taking supremum over $t \in J$ on both sides of the last inequality, then substituting the radius $r$ yields $\|W(z)\|_{\infty} \leqslant r$. Now, let $t_{1}$ and $t_{2} \in J$, such that $t_{1}<t_{2}$.

$$
\begin{aligned}
& \left|W(z)\left(t_{2}\right)-W(z)\left(t_{1}\right)\right| \\
& \leqslant \frac{\rho^{-\beta}}{\Gamma(\beta)} \sum_{i=1}^{N} \int_{0}^{t_{1}}\left[\left(t_{1}^{\rho}-\zeta^{\rho}\right)^{\beta-1}-\left(t_{2}^{\rho}-\zeta^{\rho}\right)^{\beta-1}\right]\left|g_{i}\left(\zeta, \mathcal{L}(z)(\zeta), \int_{0}^{\Phi(\zeta)} h(s, z(s)) d s\right)\right| d \zeta^{\rho} \\
& \quad+\frac{\rho^{-\beta}}{\Gamma(\beta)} \sum_{i=1}^{N} \int_{t_{1}}^{t_{2}}\left(t_{2}^{\rho}-\zeta^{\rho}\right)^{\beta-1}\left|g_{i}\left(\zeta, \mathcal{L}(z)(\zeta), \int_{0}^{\Phi(\zeta)} h(s, z(s)) d s\right)\right| d \zeta^{\rho} \\
& \quad+\frac{\rho^{-\beta}}{\Gamma(\beta)} \int_{0}^{t_{1}}\left[\left(t_{1}^{\rho}-\zeta^{\rho}\right)^{\beta-1}-\left(t_{2}^{\rho}-\zeta^{\rho}\right)^{\beta-1}\right]|\Lambda(z)(\zeta)| d \zeta^{\rho}+\frac{\rho^{-\beta}}{\Gamma(\beta)} \int_{t_{1}}^{t_{2}}\left(t_{2}^{\rho}-\zeta^{\rho}\right)^{\beta-1}|\Lambda(z)(\zeta)| d \zeta^{\rho} .
\end{aligned}
$$


Using an argument similar to what we have used above implies

$$
\left|W(z)\left(t_{2}\right)-W(z)\left(t_{1}\right)\right| \leqslant \frac{\rho^{-\beta}\left[\eta r+v_{1} \vartheta_{1} N T\right]}{\Gamma(1+\beta)}\left[t_{1}^{\rho \beta}-\left(t_{2}^{\rho}-t_{1}^{\rho}\right)^{\beta}-t_{2}^{\rho \beta}+\left(t_{2}^{\rho}-t_{1}^{\rho}\right)^{\beta}\right] .
$$

Using the inequality $\left|t^{\rho \beta}-s^{\rho \beta}\right| \leqslant \rho \beta t^{\rho \beta-1}|t-s|$, where $\rho \beta>1, t \geqslant s>0$, see [19], implies

$$
\left|W(z)\left(t_{2}\right)-W(z)\left(t_{1}\right)\right| \leqslant \frac{\rho^{-\beta}\left[\eta r+v_{1} \vartheta_{1} N T\right]}{\Gamma(1+\beta)} \rho \beta T^{\rho \beta-1}\left|t_{2}-t_{1}\right| \longrightarrow 0 \text { as } t_{2} \longrightarrow t_{1} .
$$

So, $W(z)(t) \in C(J, \mathbb{R}), \forall t \in J$. Therefore, the operator $W$ is a self-operator of the set $S_{r}$.

Step 2 in the proof is to show the operator $W$ is completely continuous (i.e. to prove that $W$ is a continuous operator and the set $W\left(S_{r}\right)$ is compact) as follows. Suppose the sequence $\left(z_{n}\right)_{n=1}^{\infty}$ with $z_{\mathrm{n}} \in \mathrm{S}_{\mathrm{r}}$ and let $z_{\mathrm{n}} \rightarrow z \in \mathrm{S}_{\mathrm{r}}$ when $\mathrm{n} \rightarrow \infty$.

$$
\begin{aligned}
\left|W\left(z_{\mathfrak{n}}\right)(t)-W(z)(t)\right| \leqslant & \frac{\rho^{-\beta}}{\Gamma(\beta)} \int_{0}^{t}\left(t^{\rho}-\zeta^{\rho}\right)^{\beta-1}\left|\Lambda\left(z_{\mathfrak{n}}\right)(\zeta)-\Lambda(z)(\zeta)\right| d \zeta^{\rho} \\
& +\frac{\rho^{-\beta}}{\Gamma(\beta)} \sum_{i=1}^{N} \int_{0}^{t}\left(t^{\rho}-\zeta^{\rho}\right)^{\beta-1} \mid g_{i}\left(\zeta, \mathcal{L}\left(z_{\mathfrak{n}}\right)(\zeta), \int_{0}^{\Phi(\zeta)} h\left(s, z_{\mathfrak{n}}(s)\right) d s\right) \\
& -g_{i}\left(\zeta, \mathcal{L}(z)(\zeta), \int_{0}^{\Phi(\zeta)} h(s, z(s)) d s\right) \mid d \zeta^{\rho} .
\end{aligned}
$$

Since the operator $\Lambda$ is continuous, therefore $\lim _{n \rightarrow \infty} z_{n}=z$ implies $\lim _{n \rightarrow \infty} \Lambda\left(z_{n}\right)=\Lambda(z)$. Also, $\lim _{n \rightarrow \infty} \mathcal{L}\left(z_{n}\right)=\mathcal{L}(z)$ because the operator $\mathcal{L}$ is continuous as well. From condition V1a, the function $g_{i}(t, y, z)$ is continuous in $y$ and $z \forall i \in\{1,2, \ldots, N\}$ and for almost all $t \in J$. The function $h(t, z)$ is continuous in $z$ for almost all $t \in J$ from condition V $2 a$ as well. Now conditions V1 and V2 allow us to use the dominated convergence theorem due to Lebesgue as follows,

$$
\begin{aligned}
& \lim _{n \rightarrow \infty} \int_{0}^{t}\left(t^{\rho}-\zeta^{\rho}\right)^{\beta-1}\left|\Lambda\left(z_{n}\right)(\zeta)-\Lambda(z)(\zeta)\right| d \zeta^{\rho}=\int_{0}^{t}\left(t^{\rho}-\zeta^{\rho}\right)^{\beta-1} \lim _{n \rightarrow \infty}\left|\Lambda\left(z_{n}\right)(\zeta)-\Lambda(z)(\zeta)\right| d \zeta^{\rho}=0 \\
& \lim _{n \rightarrow \infty} \int_{0}^{\Phi(\zeta)} h\left(s, z_{n}(s)\right) d s=\int_{0}^{\Phi(\zeta)} \lim _{n \rightarrow \infty} h\left(s, z_{n}(s)\right) d s=\int_{0}^{\Phi(\zeta)} h(s, z(s)) d s \\
& \int_{0}^{t}\left(t^{\rho}-\zeta^{\rho}\right)^{\beta-1} \lim _{n \rightarrow \infty}\left|g_{i}\left(\zeta, \mathcal{L}\left(z_{n}\right)(\zeta), \int_{0}^{\Phi(\zeta)} h\left(s, z_{n}(s)\right) d s\right)-g_{i}\left(\zeta, \mathcal{L}(z)(\zeta), \int_{0}^{\Phi(\zeta)} h(s, z(s)) d s\right)\right| d \zeta^{\rho}=0
\end{aligned}
$$

From Eqs. above, we have $\lim _{n \rightarrow \infty} W\left(z_{n}\right)=W(z)$ and so, the operator $W$ is continuous. Suppose the sequence $\left(W\left(z_{n}\right)\right)_{n=1}^{\infty}$ with $z_{n} \in S_{r}$. It is easy to see that $\left(W\left(z_{n}\right)\right)_{n=1}^{\infty}$ is uniformaly bounded because $\forall \mathrm{n} \in \mathbb{N}$ and $\forall \mathrm{t} \in \mathrm{J}$ we have $\left\|\mathrm{W}\left(z_{\mathrm{n}}\right)\right\|_{\infty} \leqslant \mathrm{r}$. Also, $\forall \mathrm{n} \in \mathbb{N}$, we have

$$
\left\|W\left(z_{n}\right)\left(t_{2}\right)-W\left(z_{n}\right)\left(t_{1}\right)\right\|_{\infty} \leqslant \frac{\rho^{-\beta}\left[\eta r+v_{1} \vartheta_{1} N T\right]}{\Gamma(1+\beta)} \rho \beta T^{\rho \beta-1}\left|t_{2}-t_{1}\right| \longrightarrow 0 \text { as } t_{2} \longrightarrow t_{1}
$$

which proves the sequence of continuous functions $\left(W\left(z_{n}\right)\right)_{n=1}^{\infty}$ is equicontinuous. From the theorem of the Ascoli-Arzela, there exists a uniformly convergent sub-sequence $\left(W\left(z_{\mathfrak{n}_{k}}\right)\right)_{\mathfrak{n}_{k}=1}^{\infty}$ in $\left(W\left(z_{\mathfrak{n}}\right)\right)_{n=1}^{\infty}$ and hence the set $W\left(S_{r}\right)$ is compact. Henceforth, the operator $W$ is completely continuous. Applying the fixed point principle due to Schauder's theorem [1] implies the existence of at least one fixed point to Eq. (2.2) and hence the existence of continuous solution in $S_{\mathrm{r}}$ to the integral equation (2.1). The proof is verified. 


\section{The infinite point non-classical condition}

In this section, we shall study the existence of mild solutions for the integro-differential equation (1.3) subject to the infinite point non-classical condition (1.5) as follows. Let the infinite series of numbers $\sum_{k=1}^{\infty} b_{k}=\lim _{n \rightarrow \infty} \sum_{k=1}^{m} b_{k}$ be convergent. Since $\left|b_{k} z\left(\tau_{k}\right)\right|=\left|b_{k}\left\|z\left(\tau_{k}\right) \mid \leqslant b_{k}\right\| z \|_{\infty}\right.$, so $\sum_{k=1}^{\infty} b_{k} z\left(\tau_{k}\right)$ is convergent, where $\tau_{k} \in J$, from applying the Weierstrass M-test. So, the condition $\sum_{k=1}^{\infty} b_{k} z\left(\tau_{k}\right)=z_{0}$ makes sense. From Eq. (2.1), we have

$$
\begin{aligned}
& z_{m}(t)=\frac{z_{0}}{\sum_{k=1}^{m} b_{k}}-\frac{\rho^{-\beta}}{\Gamma(\beta) \sum_{k=1}^{m} b_{k}}\left[\sum_{k=1}^{m} b_{k} \int_{0}^{\tau_{k}}\left(\tau_{k}^{\rho}-\zeta^{\rho}\right)^{\beta-1} \Lambda(z)(\zeta) d \zeta^{\rho}\right. \\
& \left.+\sum_{k=1}^{m} \sum_{i=1}^{N} b_{k} \int_{0}^{\tau_{k}}\left(\tau_{k}^{\rho}-\zeta^{\rho}\right)^{\beta-1} g_{i}\left(\zeta, \mathcal{L}(z)(\zeta), \int_{0}^{\Phi(\zeta)} h(s, z(s)) d s\right) d \zeta^{\rho}\right] \\
& +\frac{\rho^{-\beta}}{\Gamma(\beta)} \sum_{i=1}^{N} \int_{0}^{t}\left(t^{\rho}-\zeta^{\rho}\right)^{\beta-1} g_{i}\left(\zeta, \mathcal{L}\left(z_{m}\right)(\zeta), \int_{0}^{\Phi(\zeta)} h\left(s, z_{m}(s)\right) d s\right) d \zeta^{\rho} \\
& +\frac{\rho^{-\beta}}{\Gamma(\beta)} \int_{0}^{t}\left(t^{\rho}-\zeta^{\rho}\right)^{\beta-1} \Lambda\left(z_{m}\right)(\zeta) d \zeta^{\rho} .
\end{aligned}
$$

Passing the limit to both sides of the above equation when $m \longrightarrow \infty$ yields

$$
\begin{aligned}
\lim _{m \rightarrow \infty} z_{m}(t)= & \frac{z_{0}}{\sum_{k=1}^{\infty} b_{k}}-\frac{\rho^{-\beta}}{\Gamma(\beta) \sum_{k=1}^{\infty} b_{k}}\left[\sum_{k=1}^{\infty} b_{k} \int_{0}^{\tau_{k}}\left(\tau_{k}^{\rho}-\zeta^{\rho}\right)^{\beta-1} \Lambda(z)(\zeta) d \zeta^{\rho}\right. \\
& \left.+\sum_{k=1}^{\infty} \sum_{i=1}^{N} b_{k} \int_{0}^{\tau_{k}}\left(\tau_{k}^{\rho}-\zeta^{\rho}\right)^{\beta-1} g_{i}\left(\zeta, \mathcal{L}(z)(\zeta), \int_{0}^{\Phi(\zeta)} h(s, z(s)) d s\right) d \zeta^{\rho}\right] \\
& +\frac{\rho^{-\beta}}{\Gamma(\beta)} \sum_{i=1}^{N} \lim _{m \rightarrow \infty} \int_{0}^{t}\left(t^{\rho}-\zeta^{\rho}\right)^{\beta-1} g_{i}\left(\zeta, \mathcal{L}\left(z_{m}\right)(\zeta), \int_{0}^{\Phi(\zeta)} h\left(s, z_{m}(s)\right) d s\right) d \zeta^{\rho} \\
& +\frac{\rho^{-\beta}}{\Gamma(\beta)} \lim _{m \rightarrow \infty} \int_{0}^{t}\left(t^{\rho}-\zeta^{\rho}\right)^{\beta-1} \Lambda\left(z_{m}\right)(\zeta) d \zeta^{\rho} .
\end{aligned}
$$

Now, it is clear from the previous arguments that

$$
\frac{\rho^{-\beta}}{\Gamma(\beta)}\left|\int_{0}^{\tau_{k}}\left(\tau_{k}^{\rho}-\zeta^{\rho}\right)^{\beta-1} \Lambda(z)(\zeta) d \zeta^{\rho}\right| \leqslant \frac{\gamma r \rho^{-\beta} T^{\rho \beta}}{\Gamma(1+\beta)}=c_{1}, \text { "say". }
$$

So, the series $\sum_{k=1}^{\infty} b_{k} \int_{0}^{\tau_{k}}\left(\tau_{k}^{\rho}-\zeta^{\rho}\right)^{\beta-1} \Lambda(z)(\zeta) d \zeta^{\rho}$ is convergent from applying the Weierstrass M-test. Also, we have

$$
\begin{aligned}
& \frac{\rho^{-\beta}}{\Gamma(\beta)}\left|\sum_{i=1}^{N} \int_{0}^{\tau_{k}}\left(\tau_{k}^{\rho}-\zeta^{\rho}\right)^{\beta-1} g_{i}\left(\zeta, \mathcal{L}(z)(\zeta), \int_{0}^{\Phi(\zeta)} h(s, z(s)) d s\right) d \zeta^{\rho}\right| \\
& \left.\leqslant N v_{2}+\frac{\rho^{-\beta} N v_{1}}{\Gamma(1+\beta)}\left[\left(\gamma+\vartheta_{2} T\right) r+\vartheta_{1} T\right]\right)=c_{2}, \text { "say". }
\end{aligned}
$$

So, the series $\sum_{k=1}^{\infty} \sum_{i=1}^{N} b_{k} \int_{0}^{\tau_{k}}\left(\tau_{k}^{\rho}-\zeta^{\rho}\right)^{\beta-1} g_{i}\left(\zeta, \mathcal{L}(z)(\zeta), \int_{0}^{\Phi(\zeta)} h(s, z(s)) d s\right) d \zeta^{\rho}$ is convergent from applying the Weierstrass M-test. Now, from the Lebesgue's dominated convergence theorem, we have 


$$
\begin{aligned}
z(t)= & \frac{z_{0}}{\sum_{k=1}^{\infty} b_{k}}-\frac{\rho^{-\beta}}{\Gamma(\beta) \sum_{k=1}^{\infty} b_{k}}\left[\sum_{k=1}^{\infty} b_{k} \int_{0}^{\tau_{k}}\left(\tau_{k}^{\rho}-\zeta^{\rho}\right)^{\beta-1} \Lambda(z)(\zeta) d \zeta^{\rho}\right. \\
& \left.+\sum_{k=1}^{\infty} \sum_{i=1}^{N} b_{k} \int_{0}^{\tau_{k}}\left(\tau_{k}^{\rho}-\zeta^{\rho}\right)^{\beta-1} g_{i}\left(\zeta, \mathcal{L}(z)(\zeta), \int_{0}^{\Phi(\zeta)} h(s, z(s)) d s\right) d \zeta^{\rho}\right] \\
& +\frac{\rho^{-\beta}}{\Gamma(\beta)} \sum_{i=1}^{N} \int_{0}^{t}\left(t^{\rho}-\zeta^{\rho}\right)^{\beta-1} g_{i}\left(\zeta, \mathcal{L}(z)(\zeta), \int_{0}^{\Phi(\zeta)} h(s, z(s)) d s\right) d \zeta^{\rho} \\
& +\frac{\rho^{-\beta}}{\Gamma(\beta)} \int_{0}^{t}\left(t^{\rho}-\zeta^{\rho}\right)^{\beta-1} \Lambda(z)(\zeta) d \zeta^{\rho} .
\end{aligned}
$$

Equation (3.1) represents the integral representation of the non classical integro-differential Eq. (1.3) under the condition (1.5).

Definition 3.1 ([11]). A solution for the problem (1.3) under the nonlocal condition (1.5) is called a mild solution if it satisfies the integral equation (3.1).

Theorem 3.2. Let the series of numbers $\sum_{\mathrm{k}=1}^{\infty} \mathrm{b}_{\mathrm{k}}$ be convergent and suppose the hypotheses V1-V4 are fulfilled. Then the nonlocal problem (1.3)-(1.5) has at least one continuous mild solution.

Proof. From Thm. 2.2, the existence of continuous mild solutions for the nonlocal problem (1.3)-(1.4) is verified. Let $m \longrightarrow \infty$ and use $\sum_{k=1}^{\infty} b_{k}<\infty$. Then the existence of continuous mild solutions for the problem (1.3)-(1.4) implies directly to the existence of continuous mild solutions to the nonlocal problem (1.3)-(1.5). The proof is verified.

\section{The Riemann-Stieltjes non-classical condition}

In this section, we shall study the existence of mild solutions for the integro-differential equation (1.3) subject to the Riemann-Stieltjes integral condition (1.6) as follows. Suppose we have a partition, $0=t_{0}<t_{1}<\cdots<t_{k-1}<t_{k}<\cdots<t_{n}=T$, on the domain J. Let $\tau_{k} \in\left[t_{k-1}, t_{k}\right]$ and $b_{k}:=\Delta \hbar_{k}:=$ $\left[\hbar\left(t_{k}\right)-\hbar\left(t_{k-1}\right)\right]$ in the nonlocal condition (1.4), where the function $\hbar$ is assumed to be defined on J and monotonically increasing. So, we have

$$
\lim _{n \rightarrow \infty} \sum_{k=1}^{m} b_{k} z\left(\tau_{k}\right)=\lim _{n \rightarrow \infty} \sum_{k=1}^{m} z\left(\tau_{k}\right) \Delta \hbar_{k}=\int_{0}^{T} z(\tau) d \hbar(\tau)=z_{0}
$$

So, the condition $\int_{0}^{T} z(\tau) d \hbar(\tau)=z_{0}$ makes sense. Substituting $b_{k}=\Delta \hbar_{k}$ in Eq. (2.1) gives

$$
\begin{aligned}
z(t)= & \frac{z_{0}}{\sum_{k=1}^{m} \Delta \hbar_{k}}-\frac{\rho^{-\beta}}{\Gamma(\beta) \sum_{k=1}^{m} \Delta \hbar_{k}}\left[\sum_{k=1}^{m} \int_{0}^{\tau_{k}}\left(\tau_{k}^{\rho}-\zeta^{\rho}\right)^{\beta-1} \Lambda(z)(\zeta) d \zeta^{\rho} \Delta \hbar_{k}\right. \\
& \left.+\sum_{k=1}^{m} \sum_{i=1}^{N} \int_{0}^{\tau_{k}}\left(\tau_{k}^{\rho}-\zeta^{\rho}\right)^{\beta-1} g_{i}\left(\zeta, \mathcal{L}(z)(\zeta), \int_{0}^{\Phi(\zeta)} h(s, z(s)) d s\right) d \zeta^{\rho} \Delta \hbar_{k}\right] \\
& +\frac{\rho^{-\beta}}{\Gamma(\beta)} \sum_{i=1}^{N} \int_{0}^{t}\left(t^{\rho}-\zeta^{\rho}\right)^{\beta-1} g_{i}\left(\zeta, \mathcal{L}(z)(\zeta), \int_{0}^{\Phi(\zeta)} h(s, z(s)) d s\right) d \zeta^{\rho} \\
& +\frac{\rho^{-\beta}}{\Gamma(\beta)} \int_{0}^{t}\left(t^{\rho}-\zeta^{\rho}\right)^{\beta-1} \Lambda(z)(\zeta) d \zeta^{\rho} .
\end{aligned}
$$

Passing the limit to both sides when $m \longrightarrow \infty$, yields

$$
z(t)=\frac{z_{0}}{\lim _{m \rightarrow \infty} \sum_{k=1}^{m} \Delta \hbar_{k}}-\frac{\rho^{-\beta}}{\Gamma(\beta) \lim _{m \rightarrow \infty} \sum_{k=1}^{m} \Delta \hbar_{k}}\left[\lim _{m \rightarrow \infty} \sum_{k=1}^{m} \int_{0}^{\tau_{k}}\left(\tau_{k}^{\rho}-\zeta^{\rho}\right)^{\beta-1} \Lambda(z)(\zeta) d \zeta^{\rho} \Delta \hbar_{k}\right.
$$




$$
\begin{aligned}
& \left.+\lim _{m \rightarrow \infty} \sum_{k=1}^{m} \sum_{i=1}^{N} \int_{0}^{\tau_{k}}\left(\tau_{k}^{\rho}-\zeta^{\rho}\right)^{\beta-1} g_{i}\left(\zeta, \mathcal{L}(z)(\zeta), \int_{0}^{\Phi(\zeta)} h(s, z(s)) d s\right) d \zeta^{\rho} \Delta \hbar_{k}\right] \\
& +\frac{\rho^{-\beta}}{\Gamma(\beta)} \sum_{i=1}^{N} \int_{0}^{t}\left(t^{\rho}-\zeta^{\rho}\right)^{\beta-1} g_{i}\left(\zeta, \mathcal{L}(z)(\zeta), \int_{0}^{\Phi(\zeta)} h(s, z(s)) d s\right) d \zeta^{\rho} \\
& +\frac{\rho^{-\beta}}{\Gamma(\beta)} \int_{0}^{t}\left(t^{\rho}-\zeta^{\rho}\right)^{\beta-1} \Lambda(z)(\zeta) d \zeta^{\rho} .
\end{aligned}
$$

Applying the Riemann-Stieltjes definition of integration implies

$$
\begin{aligned}
z(t)= & \frac{z_{0}}{[\hbar(T)-\hbar(0)]} \frac{-\rho^{-\beta}}{\Gamma(\beta)[\hbar(T)-\hbar(0)]}\left[\int_{0}^{T} \int_{0}^{t}\left(t^{\rho}-\zeta^{\rho}\right)^{\beta-1} \Lambda(z)(\zeta) d \zeta^{\rho} d \hbar(t)\right. \\
& \left.+\sum_{i=1}^{N} \int_{0}^{T} \int_{0}^{t}\left(t^{\rho}-\zeta^{\rho}\right)^{\beta-1} g_{i}\left(\zeta, \mathcal{L}(z)(\zeta), \int_{0}^{\Phi(\zeta)} h(s, z(s)) d s\right) d \zeta^{\rho} d \hbar(t)\right] \\
& +\frac{\rho^{-\beta}}{\Gamma(\beta)} \sum_{i=1}^{N} \int_{0}^{t}\left(t^{\rho}-\zeta^{\rho}\right)^{\beta-1} g_{i}\left(\zeta, \mathcal{L}(z)(\zeta), \int_{0}^{\Phi(\zeta)} h(s, z(s)) d s\right) d \zeta^{\rho} \\
& +\frac{\rho^{-\beta}}{\Gamma(\beta)} \int_{0}^{t}\left(t^{\rho}-\zeta^{\rho}\right)^{\beta-1} \Lambda(z)(\zeta) d \zeta^{\rho} .
\end{aligned}
$$

Equation (4.1) represents the integral representation of the non classical integro-differential problem (1.3) under the condition (1.6).

Definition 4.1 ([11]). A solution to the nonlocal problem (1.3)-(1.6) is called a mild solution if it satisfies the integral equation (4.1).

Theorem 4.2. Let the function $\hbar$ be a monotonically increasing function defined on $\mathrm{J}$ with values in $\mathbb{R}$. Let $b_{k}:=\Delta \hbar_{k}$ in the condition (1.4). Suppose the hypotheses V1-V4 are fulfilled. Then the nonlocal problem (1.3)-(1.6) has at least one continuous mild solution.

Proof. From Thm. (2.2), the existence of continuous mild solutions of the nonlocal problem (1.3)-(1.4) is verified. Let $b_{k}:=\Delta \hbar_{k}:=\left[\hbar_{k}\left(t_{k}\right)-\hbar\left(t_{k-1}\right)\right]$ in the non classical condition (1.4), where the function $\hbar$ is assumed to be defined on $J$ and monotonically increasing, and let $m \longrightarrow \infty$. Then the existence of continuous mild solutions of the problem (1.3)-(1.4) implies directly to the existence of continuous mild solutions to the nonlocal problem (1.3)-(1.6). The proof is verified.

\section{Uniqueness results}

Assume the following hypotheses are satisfied.

(V5) The mappings $g_{i}: J \times \mathbb{R}^{2} \longrightarrow \mathbb{R}, 1 \leqslant i \leqslant N<\infty$ are measurable with respect to $t, \forall(y, z) \in \mathbb{R}^{2}$ and verify the assumptions:

(a) $\left|g_{i}\left(t, y_{2}, z_{2}\right)-g_{i}\left(t, y_{1}, z_{1}\right)\right| \leqslant v_{1}\left|y_{2}-y_{1}\right|+v_{1}\left|z_{2}-z_{1}\right|, v_{1}>0, \forall t \in J$;

(b) $\sup _{t \in J} I_{0^{+}}^{\beta, \rho}\left|g_{i}(t, 0,0)\right| \leqslant v_{2}, \forall 1 \leqslant i \leqslant N<\infty, v_{2}>0$.

(V6) The mapping $h: J \times \mathbb{R} \longrightarrow \mathbb{R}_{>0}$ is measurable with respect to $t, \forall z \in \mathbb{R}$ and satisfies the conditions:

(a) $\left|h\left(t, z_{2}\right)-h\left(t, z_{1}\right)\right| \leqslant \vartheta_{2}\left|z_{2}-z_{1}\right|, \vartheta_{2}>0$;

(b) $|\mathrm{h}(\mathrm{t}, 0)| \leqslant \vartheta_{1}, \vartheta_{1}>0, \forall \mathrm{t} \in \mathrm{J}$.

Theorem 5.1. Assume the conditions V3 - V6 are verified. Then the nonlocal problem (1.3)-1.4 has a unique continuous mild solution. 
Proof. It is clear that condition V5 implies condition V1, because $\forall 1 \leqslant i \leqslant N<\infty$ we have

$$
\left|g_{\mathfrak{i}}(t, y, z)-g_{\mathfrak{i}}(t, 0,0)+g_{\mathfrak{i}}(t, 0,0)\right| \leqslant\left|g_{\mathfrak{i}}(t, 0,0)\right|+\left|g_{\mathfrak{i}}(t, y, z)-g_{\mathfrak{i}}(t, 0,0)\right| \leqslant\left|g_{\mathfrak{i}}(t, 0,0)\right|+v_{1}|y|+v_{1}|z| .
$$

Also, condition V6 implies condition V2. So, the mild solution exists from Thm. 2.2. Let $y$ and $z$ be two mild solutions of the considered problem (1.3)-(1.4),

$$
\begin{aligned}
|z(t)-y(t)| \leqslant & \frac{A^{-1} \rho^{-\beta}}{\Gamma(\beta)} \sum_{k=1}^{m} b_{k} \int_{0}^{\tau_{k}}\left(\tau_{k}^{\rho}-\zeta^{\rho}\right)^{\beta-1}|\Lambda(z-y)(\zeta)| d \zeta^{\rho} \\
& +\frac{A^{-1} \rho^{-\beta}}{\Gamma(\beta)} \sum_{k=1}^{m} \sum_{i=1}^{N} b_{k} \int_{0}^{\tau_{k}}\left(\tau_{k}^{\rho}-\zeta^{\rho}\right)^{\beta-1} \mid g_{i}\left(\zeta, \mathcal{L}(z)(\zeta), \int_{0}^{\Phi(\zeta)} h(s, z(s)) d s\right) \\
& -g_{i}\left(\zeta, \mathcal{L}(y)(\zeta), \int_{0}^{\Phi(\zeta)} h(s, y(s)) d s\right) \mid d \zeta^{\rho} \\
& +\frac{\rho^{-\beta}}{\Gamma(\beta)} \sum_{i=1}^{N} \int_{0}^{t}\left(t^{\rho}-\zeta^{\rho}\right)^{\beta-1} \mid g_{i}\left(\zeta, \mathcal{L}(z)(\zeta), \int_{0}^{\Phi(\zeta)} h(s, z(s)) d s\right) \\
& -g_{i}\left(\zeta, \mathcal{L}(y)(\zeta), \int_{0}^{\Phi(\zeta)} h(s, y(s)) d s\right)\left|d \zeta^{\rho}+\frac{\rho^{-\beta}}{\Gamma(\beta)} \int_{0}^{t}\left(t^{\rho}-\zeta^{\rho}\right)^{\beta-1}\right| \Lambda(z-y)(\zeta) \mid d \zeta^{\rho} .
\end{aligned}
$$

Applying conditions V5a and V6a yields

$$
\begin{aligned}
|z(t)-y(t)| \leqslant & \frac{A^{-1} \gamma \rho^{-\beta}\|z-y\|_{\infty}}{\Gamma(\beta)} \sum_{k=1}^{m} b_{k} \int_{0}^{\tau_{k}}\left(\tau_{k}^{\rho}-\zeta^{\rho}\right)^{\beta-1} d \zeta^{\rho} \\
& +\frac{A^{-1} N \rho^{-\beta}}{\Gamma(\beta)} \sum_{k=1}^{m} b_{k} \int_{0}^{\tau_{k}}\left(\tau_{k}^{\rho}-\zeta^{\rho}\right)^{\beta-1}\left[v_{1}|\mathcal{L}(z-y)(\zeta)|+v_{1} \vartheta_{2} \int_{0}^{\Phi(\zeta)}|z(s)-y(s)| d s\right] d \zeta^{\rho} \\
& +\frac{N \rho^{-\beta}}{\Gamma(\beta)} \int_{0}^{t}\left(t^{\rho}-\zeta^{\rho}\right)^{\beta-1}\left[v_{1}|\mathcal{L}(z-y)(\zeta)|+v_{1} \vartheta_{2} \int_{0}^{\Phi(\zeta)}|z(s)-y(s)| d s\right] d \zeta^{\rho} \\
& +\frac{\gamma \rho^{-\beta}\|z-y\|_{\infty}}{\Gamma(\beta)} \int_{0}^{t}\left(t^{\rho}-\zeta^{\rho}\right)^{\beta-1} d \zeta^{\rho} .
\end{aligned}
$$

Simplifying, then using condition $\mathrm{V} 3$ gives

$$
|z(t)-y(t)| \leqslant \frac{2 \eta \rho^{-\beta} T^{\rho \beta}}{\Gamma(1+\beta)}\|z-y\|_{\infty} .
$$

Taking supremum over $t \in J$ on both sides gives

$$
\left[1-\frac{2 \eta \rho^{-\beta} T^{\rho \beta}}{\Gamma(1+\beta)}\right]\|z-y\|_{\infty} \leqslant 0
$$

but $\frac{2 \eta \rho^{-\beta} T^{\rho \beta}}{\Gamma(1+\beta)}<1$ from condition V3. So, $\|z-y\|_{\infty}=0$ and hence $z(t)=y(t) \forall t \in J$ which implies the uniqueness of the mild solution. The proof is verified.

Corollary 5.2. Let the series of numbers $\sum_{\mathrm{k}=1}^{\infty} \mathrm{b}_{\mathrm{k}}$ be convergent and suppose the hypotheses V3 - V6 are fulfilled. Then the nonlocal problem (1.3)-(1.5) has a unique continuous mild solution.

Proof. The poof follows directly from Thm. 5.1 after using the condition $\sum_{k=1}^{\infty} b_{k}<\infty$, then letting $\mathrm{m} \longrightarrow \infty$. 
Corollary 5.3. Let the function $\hbar$ be a monotonically increasing function defined on $\mathrm{J}$ with values in $\mathbb{R}$. Let $b_{k}:=\Delta \hbar_{k}$ in the condition (1.4). Suppose the hypotheses V3-V6 are fulfilled. Then the nonlocal problem (1.3)-(1.6) has a unique continuous mild solution.

Proof. The poof follows directly from Thm. 5.1 after substituting $b_{k}:=\Delta \hbar_{k}:=\left[\hbar_{k}\left(t_{k}\right)-\hbar\left(t_{k-1}\right)\right]$ in the non classical condition (1.4), then letting $\mathrm{m} \longrightarrow \infty$.

\section{Continuous dependence of solution}

Definition 6.1 ([10]). A mild solution $z\left(t ; z_{0}\right), t \in J$, of Eq. (1.3) under the condition (1.4) depends continuously on $z_{0}$ if $\forall \epsilon>0, \exists \delta>0$, where $\delta=\delta(\epsilon)$, such that $\left\|z-z^{*}\right\|_{\infty}<\epsilon$, whenever $\left|z_{0}-z_{0}^{*}\right|<\delta$, where $z^{*}:=z^{*}\left(t ; z_{0}^{*}\right)$ is the mild solution associated to the problem

$$
\left({ }^{C} D_{0^{+}}^{\beta, \rho}-\Lambda\right) z^{*}(t)=\sum_{i=1}^{N} g_{i}\left(t, \mathcal{L}\left(z^{*}\right)(t), \int_{0}^{\Phi(t)} h\left(\tau, z^{*}(\tau)\right) d \tau\right), \quad \text { a.e. } t \in(0, T],
$$

subject to the condition

$$
\sum_{k=1}^{m} b_{k} z^{*}\left(\tau_{k}\right)=z_{0}^{*}, \quad b_{k}>0, \tau_{k} \in(0, T] .
$$

Theorem 6.2. Suppose the hypotheses V3-V6 are fulfilled. Then the mild solution of Eq. (1.3) under the condition (1.4) depends continuously on $z_{0}$.

Proof. Let $z(\mathrm{t}):=z\left(\mathrm{t} ; z_{0}\right)$ and $z^{*}(\mathrm{t}):=z^{*}\left(\mathrm{t} ; z_{0}^{*}\right)$ be two solutions for the problems (1.3)-(1.4), and (6.1)-(6.2), respectively, where $\left|z_{0}-z_{0}^{*}\right|<\delta$. Then

$$
\begin{aligned}
\left|z(t)-z^{*}(t)\right| \leqslant & A^{-1}\left|z_{0}-z_{0}^{*}\right|+A^{-1} \frac{\rho^{-\beta}}{\Gamma(\beta)} \sum_{k=1}^{m} b_{k} \int_{0}^{\tau_{k}}\left(\tau_{k}^{\rho}-\zeta^{\rho}\right)^{\beta-1}\left|\Lambda\left(z-z^{*}\right)(\zeta)\right| d \zeta^{\rho} \\
& +\frac{A^{-1} \rho^{-\beta}}{\Gamma(\beta)} \sum_{k=1}^{m} \sum_{i=1}^{N} b_{k} \int_{0}^{\tau_{k}}\left(\tau_{k}^{\rho}-\zeta^{\rho}\right)^{\beta-1} \mid g_{i}\left(\zeta, \mathcal{L}(z)(\zeta), \int_{0}^{\Phi(\zeta)} h(s, z(s)) d s\right) \\
& -g_{i}\left(\zeta, \mathcal{L}\left(z^{*}\right)(\zeta), \int_{0}^{\Phi(\zeta)} h\left(s, z^{*}(s)\right) d s\right) \mid d \zeta^{\rho} \\
& +\frac{\rho^{-\beta}}{\Gamma(\beta)} \sum_{i=1}^{N} \int_{0}^{t}\left(t^{\rho}-\zeta^{\rho}\right)^{\beta-1} \mid g_{i}\left(\zeta, \mathcal{L}(z)(\zeta), \int_{0}^{\Phi(\zeta)} h(s, z(s)) d s\right) \\
& -g_{i}\left(\zeta, \mathcal{L}\left(z^{*}\right)(\zeta), \int_{0}^{\Phi(\zeta)} h\left(s, z^{*}(s)\right) d s\right)\left|d \zeta^{\rho}+\frac{\rho^{-\beta}}{\Gamma(\beta)} \int_{0}^{t}\left(t^{\rho}-\zeta^{\rho}\right)^{\beta-1}\right| \Lambda\left(z-z^{*}\right)(\zeta) \mid d \zeta^{\rho} .
\end{aligned}
$$

So, we have

$$
\left|z(t)-z^{*}(t)\right| \leqslant A^{-1} \delta+\frac{2 \eta \rho^{-\beta} T^{\rho \beta}}{\Gamma(1+\beta)}\left\|z-z^{*}\right\|_{\infty} .
$$

Taking supremum over $t \in J$ on both sides yields $\left\|z-z^{*}\right\|_{\infty} \leqslant \frac{\Gamma(1+\beta) A^{-1}}{\Gamma(1+\beta)-2 \eta \rho^{-\beta} T^{\rho \beta}} \delta$. Let $\delta=\frac{\Gamma(1+\beta)-2 \eta \rho^{-\beta} T^{\rho \beta}}{\Gamma(1+\beta) A^{-1}} \epsilon$. Therefore, $\left\|z-z^{*}\right\|_{\infty} \leqslant \epsilon$. The proof is verified.

Definition 6.3 ([10]). A mild solution $z(t ; h), t \in J$, of Eq. (1.3) under the condition (1.4) depends continuously on the function $h$ if $\forall \epsilon>0, \exists \delta>0$, where $\delta=\delta(\epsilon)$, such that $\left\|z-z^{*}\right\|_{\infty}<\epsilon$, whenever $\left|h-h^{*}\right|<\delta$, where $h^{*}:=h^{*}\left(t, z^{*}\right)$ and $z^{*}:=z^{*}\left(t ; h^{*}\right)$ is the mild solution associated to the problem

$$
\left({ }^{C} D_{0^{+}}^{\beta, \rho}-\Lambda\right) z^{*}(t)=\sum_{i=1}^{N} g_{i}\left(t, \mathcal{L}\left(z^{*}\right)(t), \int_{0}^{\Phi(t)} h^{*}\left(\tau, z^{*}(\tau)\right) d \tau\right), \quad \text { a.e. } t \in(0, T],
$$


subject to the condition

$$
\sum_{k=1}^{m} b_{k} z^{*}\left(\tau_{k}\right)=z_{0}, \quad b_{k}>0, \tau_{k} \in(0, T] .
$$

Theorem 6.4. Suppose the hypotheses V3-V6 are fulfilled. Then the mild solution of Eq. (1.3) under the condition (1.4) depends continuously on the function $\mathrm{h}$.

Proof. Let $z(\mathrm{t}):=z(\mathrm{t} ; \mathrm{h})$ and $z^{*}(\mathrm{t}):=z^{*}\left(\mathrm{t} ; \mathrm{h}^{*}\right)$ be two solutions of the problems (1.3)-(1.4) and (6.3)-(6.4), respectively, where $\left|h-h^{*}\right|<\delta$. Then

$$
\begin{aligned}
\left|z(t)-z^{*}(t)\right| \leqslant & \frac{A^{-1} \rho^{-\beta}}{\Gamma(\beta)} \sum_{k=1}^{m} b_{k} \int_{0}^{\tau_{k}}\left(\tau_{k}^{\rho}-\zeta^{\rho}\right)^{\beta-1}\left|\Lambda\left(z-z^{*}\right)(\zeta)\right| d \zeta^{\rho} \\
& +\frac{A^{-1} \rho^{-\beta}}{\Gamma(\beta)} \sum_{k=1}^{m} \sum_{i=1}^{N} b_{k} \int_{0}^{\tau_{k}}\left(\tau_{k}^{\rho}-\zeta^{\rho}\right)^{\beta-1} \mid g_{i}\left(\zeta, \mathcal{L}(z)(\zeta), \int_{0}^{\Phi(\zeta)} h(s, z(s)) d s\right) \\
& -g_{i}\left(\zeta, \mathcal{L}\left(z^{*}\right)(\zeta), \int_{0}^{\Phi(\zeta)} h^{*}\left(s, z^{*}(s)\right) d s\right) \mid d \zeta^{\rho} \\
& +\frac{\rho^{-\beta}}{\Gamma(\beta)} \sum_{i=1}^{N} \int_{0}^{t}\left(t^{\rho}-\zeta^{\rho}\right)^{\beta-1} \mid g_{i}\left(\zeta, \mathcal{L}(z)(\zeta), \int_{0}^{\Phi(\zeta)} h(s, z(s)) d s\right) \\
& -g_{i}\left(\zeta, \mathcal{L}\left(z^{*}\right)(\zeta), \int_{0}^{\Phi(\zeta)} h^{*}\left(s, z^{*}(s)\right) d s\right)\left|d \zeta^{\rho}+\frac{\rho^{-\beta}}{\Gamma(\beta)} \int_{0}^{t}\left(t^{\rho}-\zeta^{\rho}\right)^{\beta-1}\right| \Lambda\left(z-z^{*}\right)(\zeta) \mid d \zeta^{\rho} .
\end{aligned}
$$

Now, we have

$$
\begin{aligned}
\left|z(\mathrm{t})-z^{*}(\mathrm{t})\right| \leqslant & \frac{2 \gamma \rho^{-\beta} T^{\rho \beta}\left(1+N v_{1}\right)}{\Gamma(1+\beta)}\left\|z-z^{*}\right\|_{\infty} \\
& +\frac{A^{-1} N v_{1} \rho^{-\beta}}{\Gamma(\beta)} \sum_{k=1}^{m} b_{k} \int_{0}^{\tau_{k}} \int_{0}^{\Phi(\zeta)}\left(\tau_{k}^{\rho}-\zeta^{\rho}\right)^{\beta-1}\left|h(s, z(s))-h\left(s, z^{*}(s)\right)\right| \mathrm{dsd} \zeta^{\rho} \\
& +\frac{A^{-1} N v_{1} \rho^{-\beta}}{\Gamma(\beta)} \sum_{k=1}^{m} b_{k} \int_{0}^{\tau_{k}} \int_{0}^{\Phi(\zeta)}\left(\tau_{k}^{\rho}-\zeta^{\rho}\right)^{\beta-1}\left|h\left(s, z^{*}(s)\right)-h^{*}\left(s, z^{*}(s)\right)\right| \mathrm{d} s \mathrm{~d} \zeta^{\rho} \\
& +\frac{N v_{1} \rho^{-\beta}}{\Gamma(\beta)} \int_{0}^{t} \int_{0}^{\Phi(\zeta)}\left(t^{\rho}-\zeta^{\rho}\right)^{\beta-1}\left|h(s, z(s))-h\left(s, z^{*}(s)\right)\right| d s d \zeta^{\rho} \\
& +\frac{N v_{1} \rho^{-\beta}}{\Gamma(\beta)} \int_{0}^{t} \int_{0}^{\Phi(\zeta)}\left(t^{\rho}-\zeta^{\rho}\right)^{\beta-1}\left|h\left(s, z^{*}(s)\right)-h^{*}\left(s, z^{*}(s)\right)\right| \mathrm{dsd} \zeta^{\rho} .
\end{aligned}
$$

Evaluating the double integral in the above inequality and simplifying the results gives

$$
\left|z(t)-z^{*}(t)\right| \leqslant \frac{2 \eta \rho^{-\beta} T^{\rho \beta}}{\Gamma(1+\beta)}\left\|z-z^{*}\right\|_{\infty}+\frac{2 N v_{1} \rho^{-\beta} T^{(1+\rho \beta)}}{\Gamma(1+\beta)}\left|h-h^{*}\right| .
$$

Passing the supremum over $t \in J$ on both sides gives $\left\|z-z^{*}\right\|_{\infty} \leqslant \frac{2 N v_{1} \rho^{-\beta} T^{(1+\rho \beta)}}{\Gamma(1+\beta)-2 \eta \rho^{-\beta} T^{\rho \beta}} \delta$. Using $\delta=$ $\frac{\Gamma(1+\beta)-2 \eta \rho^{-\beta} T^{\rho \beta}}{2 N v_{1} \rho^{-\beta} T^{(1+\rho \beta)}} \epsilon$ implies $\left\|z-z^{*}\right\|_{\infty} \leqslant \epsilon$. The proof is verified.

Definition 6.5 ([10]). A mild solution $z\left(t ; b_{k}\right), t \in J$, of Eq. (1.3) under the condition (1.4) depends continuously on $b_{k}$ if $\forall \epsilon>0, \exists \delta>0$, where $\delta=\delta(\epsilon)$, such that $\left\|z-z^{*}\right\|_{\infty}<\epsilon$, whenever $\left|b_{k}-b_{k}^{*}\right|<\delta$, where $z^{*}:=z^{*}\left(t ; b_{k}^{*}\right)$ is the mild solution associated to the problem

$$
\left({ }^{C} D_{0^{+}}^{\beta, \rho}-\Lambda\right) z^{*}(t)=\sum_{i=1}^{N} g_{i}\left(t, \mathcal{L}\left(z^{*}\right)(t), \int_{0}^{\Phi(t)} h\left(\tau, z^{*}(\tau)\right) d \tau\right), \quad \text { a.e. } t \in(0, T],
$$


subject to the condition

$$
\sum_{k=1}^{m} b_{k}^{*} z^{*}\left(\tau_{k}\right)=z_{0}, \quad b_{k}>0, \tau_{k} \in(0, T] .
$$

Theorem 6.6. Suppose the hypotheses V3-V6 are fulfilled. Then the mild solution of Eq. (1.3) under the condition (1.4) depends continuously on $b_{k}$.

Proof. Let $z(t):=z\left(t ; b_{k}\right)$ and $z^{*}(t):=z^{*}\left(t ; b_{k}^{*}\right)$ be two solutions of the problems (1.3)-(1.4), and (6.5)-(6.6), respectively, where $\left|b_{k}-b_{k}^{*}\right|<\delta$. Then

$$
\begin{aligned}
\left|z(t)-z^{*}(t)\right| \leqslant & A^{-1} A^{*-1}\left|z_{0}\right| \sum_{k=1}^{m}\left|b_{k}-b_{k}^{*}\right|+\frac{\eta \rho^{-\beta} T^{\rho \beta}}{\Gamma(1+\beta)}\left\|z-z^{*}\right\|_{\infty} \\
& +\frac{A^{*-1} \rho^{-\beta}}{\Gamma(\beta)} \sum_{k=1}^{m} b_{k}^{*} \int_{0}^{\tau_{k}}\left(\tau_{k}^{\rho}-\zeta^{\rho}\right)^{\beta-1}\left|\Lambda\left(z-z^{*}\right)(\zeta)\right| d \zeta^{\rho} \\
& +\frac{\rho^{-\beta} A^{-1} A^{*-1}}{\Gamma(\beta)} \sum_{k=1}^{m}\left|b_{k}-b_{k}^{*}\right| \sum_{k=1}^{m} b_{k} \int_{0}^{\tau_{k}}\left(\tau_{k}^{\rho}-\zeta^{\rho}\right)^{\beta-1}|\Lambda(z)(\zeta)| d \zeta^{\rho} \\
& +\frac{A^{*-1} \rho^{-\beta}}{\Gamma(\beta)} \sum_{k=1}^{m}\left|b_{k}-b_{k}^{*}\right| \int_{0}^{\tau_{k}}\left(\tau_{k}^{\rho}-\zeta^{\rho}\right)^{\beta-1}|\Lambda(z)(\zeta)| d \zeta^{\rho} \\
& +\frac{A^{*-1} \rho^{-\beta}}{\Gamma(\beta)} \sum_{k=1}^{m} \sum_{i=1}^{N} b_{k}^{*} \int_{0}^{\tau_{k}}\left(\tau_{k}^{\rho}-\zeta^{\rho}\right)^{\beta-1} \mid g_{i}\left(\zeta, \mathcal{L}(z)(\zeta), \int_{0}^{\Phi(\zeta)} h(s, z(s)) d s\right) \\
& -g_{i}\left(\zeta, \mathcal{L}\left(z^{*}\right)(\zeta), \int_{0}^{\Phi(\zeta)} h\left(s, z^{*}(s)\right) d s\right) \mid d \zeta^{\rho}+\frac{\rho^{-\beta} A^{-1} A^{*-1}}{\Gamma(\beta)} \\
& \times \sum_{k=1}^{m}\left|b_{k}-b_{k}^{*}\right| \sum_{k=1}^{m} \sum_{i=1}^{N} b_{k} \int_{0}^{\tau_{k}}\left(\tau_{k}^{\rho}-\zeta^{\rho}\right)^{\beta-1}\left|g_{i}\left(\zeta, \mathcal{L}(z)(\zeta), \int_{0}^{\Phi(\zeta)} h(s, z(s)) d s\right)\right| d \zeta^{\rho} \\
& +\frac{A^{*-1} \rho^{-\beta}}{\Gamma(\beta)} \sum_{k=1}^{m}\left|b_{k}-b_{k}^{*}\right| \int_{0}^{\tau_{k}}\left(\tau_{k}^{\rho}-\zeta^{\rho}\right)^{\beta-1}\left|g_{i}\left(\zeta, \mathcal{L}(z)(\zeta), \int_{0}^{\Phi(\zeta)} h(s, z(s)) d s\right)\right| d \zeta^{\rho} .
\end{aligned}
$$

Simplifying the above inequality yields

$$
\begin{aligned}
\left|z(t)-z^{*}(t)\right| \leqslant & A^{-1} A^{*-1} m\left|z_{0}\right| \delta+2 N A^{*-1} v_{2} m \delta+\frac{2 \eta \rho^{-\beta} T^{\rho \beta}}{\Gamma(1+\beta)}\left\|z-z^{*}\right\|_{\infty} \\
& +\frac{2 \eta A^{*-1} \rho^{-\beta} T^{\rho \beta} m r}{\Gamma(1+\beta)} \delta+\frac{2 A^{*-1} \rho^{-\beta} T^{(1+\rho \beta)} v_{1} \vartheta_{1} m}{\Gamma(1+\beta)} \delta .
\end{aligned}
$$

Taking supremum over $t \in J$ on both sides yields

$$
\left\|z-z^{*}\right\|_{\infty} \leqslant \frac{A^{*-1} m\left[\left(A^{-1}\left|z_{0}\right|+2 N v_{2}\right) \Gamma(1+\beta)+2 \rho^{-\beta} T^{\rho \beta}\left(\eta r+v_{1} \vartheta_{1} T\right)\right]}{\Gamma(1+\beta)-2 \eta \rho^{-\beta} T^{\rho \beta}} \delta .
$$

Using $\delta=\frac{\Gamma(1+\beta)-2 \eta \rho^{-\beta} T^{\rho \beta}}{A^{*-1} m\left[\left(A^{-1}\left|z_{0}\right|+2 N v_{2}\right) \Gamma(1+\beta)+2 \rho^{-\beta} T^{\rho \beta}\left(\eta r+v_{1} \vartheta_{1} T\right)\right]} \epsilon$ implies $\left\|z-z^{*}\right\|_{\infty} \leqslant \epsilon$. The proof is verified.

\section{Illustrative examples}

In this section we present two examples to illustrate the applicability of the results in this paper. Without loss of generality, let $\mathrm{N}=1$. 
Example 7.1. Consider the following generalized fractional integro-differential equation:

$$
\begin{aligned}
& \left({ }^{\mathrm{C}} \mathrm{D}_{0^{+}}^{\frac{1}{2}, \frac{9}{2}}-\frac{1}{100} e^{-\omega t}\right) z(\mathrm{t}) \\
& \quad=\frac{1}{6} \mathrm{t}^{2}+\frac{1}{150} \int_{0}^{\mathrm{t}}(\mathrm{t}+\sin (\mathrm{s})) z(\mathrm{~s}) \mathrm{d} \mathrm{s}+\frac{1}{300} \int_{0}^{p t \mathrm{e}^{-\mathrm{t}}}\left(\frac{\mathrm{s}}{3 \mathrm{e}^{|z(\mathrm{~s})|}+(\mathrm{s}+1)^{2}}+|\ln (1+|z(\mathrm{~s})|)|\right) \mathrm{d} \mathrm{s},
\end{aligned}
$$

where, $0<p<1,0<\omega<\infty, t \in(0,1]$ and subject to the non-classical condition

$$
\sum_{k=1}^{\infty} \frac{1}{k^{6}} z\left(\frac{3 k-1}{3 k}\right)=0
$$

Comparing Eqs. (7.1)-(7.2) with the model (1.3)-(1.5) gives the following. The parameters $\beta=\frac{1}{2}, \rho=\frac{9}{2}$, $\mathrm{T}=1$ and $\rho \beta=\frac{9}{4}>1$. The lag function is $\Phi(\mathrm{t})=p t e^{-\mathrm{t}}$, because $p t e^{-t}=\mathrm{t}-\left(1-p e^{-\mathrm{t}}\right) \mathrm{t} \Longrightarrow$ the fading delay is $\varphi(t)=\left(1-p e^{-t}\right) t$ where $\varphi(0)=0$ and $0<\varphi(t)<t \forall t \in(0,1]$. It is clear that the lag function, $\Phi(t)=p t e^{-t}$, is vanishing and continuous self-map on $[0,1]$ and hence condition V4 is satisfied. The operator $\Lambda(z)(t):=\frac{1}{100} e^{-\omega t} z(t)$, where $0<\omega<\infty$. It is clear that $\Lambda$ is a linear selfoperators on $C(J, \mathbb{R})$. Let $\left(z_{n}\right)_{n=1}^{\infty}$ be a sequence with $z_{n} \in \mathcal{D}(\Lambda)$ such that $Z_{n} \longrightarrow z, \Lambda\left(Z_{n}\right) \longrightarrow y$. Now $\lim _{n \rightarrow \infty} \Lambda\left(Z_{n}\right)(t)=\lim _{n \rightarrow \infty} \frac{1}{100} e^{-\omega t} z_{n}(t)=\frac{1}{100} e^{-\omega t} \lim _{n \rightarrow \infty} z_{n}(t)=\frac{1}{100} e^{-\omega t} z(t)=\Lambda(z)(t) \Longrightarrow$ $y=\Lambda(z)$ and clearly $z \in C(J, \mathbb{R}) \Longrightarrow z \in \mathcal{D}(\Lambda)$ and hence the operator $\Lambda$ is closed. From the closed graph theorem, the operator $\Lambda$ is bounded where, $\|\Lambda(z)\|_{\infty} \leqslant \frac{1}{100}\|z\|_{\infty} \Longrightarrow \gamma_{1}=\frac{1}{100}$. The operator $\mathcal{L}(z)(t):=\int_{0}^{t} \frac{1}{150}(t+\sin (s)) z(s) d s$ and it is clear that $\mathcal{L}$ is a linear self-operator on $C(J, \mathbb{R})$. Let $\left(z_{n}\right)_{n=1}^{\infty}$ be a sequence with $z_{n} \in \mathcal{D}(\mathcal{L})$ such that $Z_{n} \longrightarrow z, \mathcal{L}\left(Z_{n}\right) \longrightarrow y$. Now $\lim _{n \rightarrow \infty} \mathcal{L}\left(Z_{n}\right)(t)=\frac{1}{150} \lim _{n \rightarrow \infty} \int_{0}^{t}(t+$ $\sin (\mathrm{s})) z_{\mathrm{n}}(\mathrm{s}) \mathrm{ds}=\frac{1}{150} \int_{0}^{\mathrm{t}}(\mathrm{t}+\sin (\mathrm{s})) \lim _{\mathrm{n} \rightarrow \infty} z_{\mathfrak{n}}(\mathrm{s}) \mathrm{d} s=\frac{1}{150} \int_{0}^{\mathrm{t}}(\mathrm{t}+\sin (\mathrm{s})) z(\mathrm{~s}) \mathrm{d} s=\mathcal{L}(z)(\mathrm{t}) \Longrightarrow \mathrm{y}=\mathcal{L}(z)$ and clearly $z \in \mathrm{C}(\mathrm{J}, \mathbb{R}) \Longrightarrow z \in \mathcal{D}(\mathcal{L})$ and hence the operator $\mathcal{L}$ is closed. From the closed graph theorem, the operator $\mathcal{L}$ is bounded where, $\|\mathcal{L}(z)\|_{\infty} \leqslant \frac{1}{75}\|z\|_{\infty} \Longrightarrow \gamma_{2}=\frac{1}{75}$ and so $\gamma:=\max \left\{\gamma_{1}, \gamma_{2}\right\}=\max \left\{\frac{1}{100}, \frac{1}{75}\right\}=$ $\frac{1}{75}$. Set the function

$$
\begin{aligned}
& g\left(t, \mathcal{L}(z)(t), \int_{0}^{\Phi(t)} h(s, z(s)) d s\right) \\
& \quad:=\frac{1}{6} t^{2}+\frac{1}{150} \int_{0}^{t}(t+\sin (s)) z(s) d s+\frac{1}{150} \int_{0}^{p t e^{-t}} \frac{1}{2}\left(\frac{s}{3 e^{|z(s)|}+(s+1)^{2}}+|\ln (1+|z(s)|)|\right) d s .
\end{aligned}
$$

It is cleat that the function $g\left(t, y_{1}, y_{2}\right)$ is measurable in $t, \forall\left(y_{1}, y_{2}\right) \in \mathbb{R}^{2}$ and continuous in $\left(y_{1}, y_{2}\right), \forall t \in J$. Also, we have

$$
\begin{aligned}
& \left|g\left(t, \mathcal{L}(z)(t), \int_{0}^{\Phi(t)} h(s, z(s)) d s\right)\right| \\
& \quad \leqslant \frac{1}{6} t^{2}+\frac{1}{150}\left|\int_{0}^{t}(t+\sin (s)) z(s) d s\right|+\frac{1}{150}\left|\int_{0}^{p t e^{-t}} \frac{1}{2}\left(\frac{s}{3 e^{|z(s)|}+(s+1)^{2}}+|\ln (1+|z(s)|)|\right) d s\right| \\
& \quad \leqslant \frac{1}{6} t^{2}+\frac{1}{150} \int_{0}^{t}(t+\sin (s))|z(s)| d s+\frac{1}{150} \int_{0}^{p t e^{-t}} \frac{1}{2}\left(\frac{s}{3 e^{|z(s)|}+(s+1)^{2}}+|\ln (1+|z(s)|)|\right) d s .
\end{aligned}
$$

So, $b(t)=\frac{1}{6} t^{2} \in L^{1}([0, T]), v_{1}=\frac{1}{150}$ and moreover, we have

$$
\sup _{t \in J} I_{0^{+}}^{\frac{1}{2}, \frac{9}{2}}\left(\frac{1}{6} t^{2}\right)=\frac{\left(\frac{9}{2}\right)^{\frac{1}{2}}}{6 \Gamma\left(\frac{1}{2}\right)} \sup _{t \in J} \int_{0}^{t} \theta^{\frac{11}{2}}\left(t^{\frac{9}{2}}-\theta^{\frac{9}{2}}\right)^{\frac{-1}{2}} d \theta=\sup _{t \in J}\left(\frac{\Gamma\left(\frac{13}{9}\right)}{9 \sqrt{2} \Gamma\left(\frac{35}{18}\right)} t^{\frac{17}{4}}\right)=\frac{\Gamma\left(\frac{13}{9}\right)}{9 \sqrt{2} \Gamma\left(\frac{35}{18}\right)}<1 .
$$


So, we can take $v_{2}=1$. Therefore, condition V1 is satisfied. Also, set the function

$$
h(t, z(t)):=\frac{1}{2}\left(\frac{t}{3 e^{|z(t)|}+(t+1)^{2}}+|\ln (1+|z(t)|)|\right),
$$

and therefore, $h(t, z)$ is positive, measurable in $t \forall z \in \mathbb{R}$ and continuous in $z, \forall t \in J$. Moreover, we have

$$
|h(t, z(t))| \leqslant \frac{1}{8}+\frac{1}{2}|z(t)| .
$$

So, we can take $\vartheta_{1}=\frac{1}{8}$ and $\vartheta_{2}=\frac{1}{2}$ and hence condition V2 is satisfied. Since $\rho^{\beta} \Gamma(1+\beta)=\left(\frac{9}{2}\right)^{\frac{1}{2}} \Gamma(1.5)=$ 1.88 , and since $2 \eta T^{\rho \beta}=2 T^{\rho \beta}\left(\gamma\left(1+v_{1} N\right)+v_{1} \vartheta_{2} N T\right)=2\left(\frac{151}{11250}+\frac{1}{300}\right)=0.034$. So, $\rho^{\beta} \Gamma(1+\beta)>2 \eta T^{\rho \beta}$ and hence condition $\mathrm{V} 3$ is satisfied. Now conditions $\mathrm{V} 1-\mathrm{V} 4$ are verified and the series of numbers $\sum_{\mathrm{k}=1}^{\infty} \frac{1}{\mathrm{k}^{6}}$ is convergent. Using Thm. 3.2, the problem (7.1)-(7.2) has at least one continuous mild solution.

Example 7.2. Assume the following generalized fractional integro-differential equation.

$$
\left({ }^{c} D_{0^{+}}^{\frac{3}{4}, \frac{8}{3}}-\frac{1}{100} e^{-\omega t}\right) z(t)=\frac{1}{4} t^{5}+\frac{1}{15} \int_{0}^{t} e^{-s} z(s) d s+\frac{1}{60} \int_{0}^{p t}\left(\frac{s}{(s+3)^{2}+5 e^{|z(s)|}}+\sin (z(s))\right) d s,
$$

where, $0<p<1,0<\omega<\infty, t \in(0,1]$ and subject to the integral condition

$$
\int_{0}^{1} s^{2} z(s) d s=\frac{1}{3}
$$

Comparing Eqs. (7.3)-(7.4) with the model (1.3)-(1.6) gives the following. The parameters $\beta=\frac{3}{4}, \rho=\frac{8}{3}$, $T=1$ and $\rho \beta=2>1$. The lag function is $\Phi(t)=p t$, because $p t=t-(1-p) t \Longrightarrow$ the fading delay is $\varphi(t)=(1-p) t$, where $\varphi(0)=0$ and $0<\varphi(t)<t \forall t \in(0,1]$. It is clear that the lag function, $\Phi(t)=p t$, is vanishing and continuous self-map on $[0,1]$ and hence condition $V 4$ is satisfied. The operator $\Lambda(z)(t):=$ $\frac{1}{100} e^{-\omega t} z(t)$, where $0<\omega<\infty$, is a linear closed self-operator on $C(J, \mathbb{R})$ and bounded as well where $\|\Lambda(z)\|_{\infty} \leqslant \frac{1}{100}\|z\|_{\infty} \Longrightarrow \gamma_{1}=\frac{1}{100}$. The operator $\mathcal{L}(z)(t):=\int_{0}^{t} \frac{1}{15} e^{-s} z(s) d s$ and it is clearly that $\mathcal{L}$ is a linear self-operators on $\mathrm{C}(\mathrm{J}, \mathbb{R})$. Let $\left(z_{\mathrm{n}}\right)_{\mathrm{n}=1}^{\infty}$ be a sequence with $z_{\mathrm{n}} \in \mathcal{D}(\mathcal{L})$ such that $Z_{\mathrm{n}} \longrightarrow z, \mathcal{L}\left(Z_{n}\right) \longrightarrow$ y. Now $\lim _{n \rightarrow \infty} \mathcal{L}\left(Z_{n}\right)(t)=\frac{1}{15} \lim _{n \rightarrow \infty} \int_{0}^{t} e^{-s} z_{n}(s) d s=\frac{1}{15} \int_{0}^{t} e^{-s} \lim _{n \rightarrow \infty} z_{n}(s) d s=\frac{1}{15} \int_{0}^{t} e^{-s} z(s) d s=$ $\mathcal{L}(z)(\mathrm{t}) \Longrightarrow \mathrm{y}=\mathcal{L}(z)$ and clearly $z \in \mathrm{C}(\mathrm{J}, \mathbb{R}) \Longrightarrow z \in \mathcal{D}(\mathcal{L})$ and hence the operator $\mathcal{L}$ is closed. From the closed graph theorem, the operator $\mathcal{L}$ is bounded, where $\|\mathcal{L}(z)\|_{\infty} \leqslant \frac{1}{15}\|z\|_{\infty} \Longrightarrow \gamma_{2}=\frac{1}{15}$ and so $\gamma:=\max \left\{\gamma_{1}, \gamma_{2}\right\}=\max \left\{\frac{1}{100}, \frac{1}{15}\right\}=\frac{1}{15}$. Set the function

$$
g\left(t, \mathcal{L}(z)(t), \int_{0}^{\Phi(t)} h(s, z(s)) d s\right):=\frac{1}{4} t^{5}+\frac{1}{15} \int_{0}^{t} e^{-s} z(s) d s+\frac{1}{60} \int_{0}^{p t}\left(\frac{s}{(s+3)^{2}+5 e^{|z(s)|}}+\sin (z(s))\right) d s .
$$

It is cleat that the function $g\left(t, y_{1}, y_{2}\right)$ is measurable in $t, \forall\left(y_{1}, y_{2}\right) \in \mathbb{R}^{2}$ and continuous in $\left(y_{1}, y_{2}\right), \forall t \in J$. Also, we have

$$
\left|g\left(t, \mathcal{L}(z)(t), \int_{0}^{\Phi(t)} h(s, z(s)) d s\right)\right| \leqslant \frac{1}{4} t^{5}+\frac{1}{15} \int_{0}^{t} e^{-s}|z(s)| d s+\frac{1}{15} \int_{0}^{p t} \frac{1}{4}\left|\frac{s}{(s+3)^{2}+5 e^{|z(s)|}}+\sin (z(s))\right| d s .
$$

So, $b(t)=\frac{1}{4} t^{5} \in \mathrm{L}^{1}([0, \mathrm{~T}]), v_{1}=\frac{1}{15}$ and moreover, we have

$$
\sup _{t \in J} I_{0^{+}}^{\frac{3}{4}, \frac{8}{3}}\left(\frac{1}{4} t^{5}\right)=\frac{\left(\frac{8}{3}\right)^{\frac{1}{4}}}{4 \Gamma\left(\frac{3}{4}\right)} \sup _{t \in J} \int_{0}^{t} \theta^{\frac{20}{3}}\left(t^{\frac{8}{3}}-\theta^{\frac{8}{3}}\right)^{\frac{-1}{4}} d \theta=\sup _{t \in J}\left(\frac{3^{\frac{3}{4}} \Gamma\left(\frac{23}{8}\right)}{2^{\frac{17}{4}} \Gamma\left(\frac{29}{8}\right)} t^{7}\right)=\left(\frac{3^{\frac{3}{4}} \Gamma\left(\frac{23}{8}\right)}{2^{\frac{17}{4}} \Gamma\left(\frac{29}{8}\right)}\right)<1 .
$$

So, we can take $v_{2}=1$. Therefore, condition V1 is satisfied. Also, set the function

$$
h(t, z(t)):=\frac{1}{4}\left(\frac{s}{(s+3)^{2}+5 e^{|z(s)|}}+\sin (z(s))\right)
$$


and therefore, $h(t, z)$ is positive, measurable in $t \forall z \in \mathbb{R}$ and continuous in $z \forall t \in J$. Also, we have

$$
|h(t, z(t))| \leqslant \frac{1}{56}+\frac{1}{4}|z(t)|
$$

So, we can take $\vartheta_{1}=\frac{1}{56}$ and $\vartheta_{2}=\frac{1}{4}$ and hence condition V2 is satisfied. Since $\rho^{\beta} \Gamma(1+\beta)=\left(\frac{8}{3}\right)^{\frac{3}{4}} \Gamma(1.75)=$ 1.918 and since $2 \eta T^{\rho \beta}=2 T^{\rho \beta}\left(\gamma\left(1+v_{1} N\right)+v_{1} \vartheta_{2} N T\right)=2\left(\frac{16}{225}+\frac{1}{60}\right)=0.176$. So, $\rho^{\beta} \Gamma(1+\beta)>2 \eta T^{\rho \beta}$ and hence condition V3 is satisfied. Now conditions V1-V4 are verified and the integrator, $\hbar(t)=t^{3}$, is monotonically increasing on J. Using Thm. 4.2, the problem (7.3)-(7.4) has at least one continuous mild solution.

\section{Conclusion}

In this work, we studied the existence and continuous dependence of solution for a generalized fractional model of integro-differential functional equation with a vanishing delay in the functional part. Our results and illustrative examples present an extending to some new results published recently by El-Sayed et al. in [10]. So, we do believe that this work is important. As a continuation to our work, we suggest studying numerical solution to this model and compare the rate of convergence using some relevant algorithms to get the most relevant one.

\section{Acknowledgment}

I would like to thank professor M. A. Abdou for his valuable comments during preparing this work.

\section{References}

[1] R. P. Agarwal, M. Meehan, D. O'Regan, Fixed Point Theory and Applications, Cambridge University Press, Cambridge, (2001). 2

[2] R. Almeida, A. B. Malinowska, T. Odzijewicz, Fractional differential equations with dependence on the CaputoKatugampola derivative, J. Comput. Nonlinear Dynam., 11 (2016), 11 pages. 1

[3] A. T. Assanova, On the theory of nonlocal problems with integral conditions for systems of equations of hyperbolic type, Ukrainian Math. J., 70 (2019), 1514-1525. 1

[4] T. M. Atanacković, S. Pilipović, B. Stanković, D. Zorica, Fractional Calculus with Applications in Mechanics: Vibrations and Diffusion Processes, John Wiley \& Sons, Hoboken, (2014). 1

[5] G. Avalishvili, M. Avalishvili, D. Gordeziani, On a nonlocal problem with integral boundary conditions for a multidimensional elliptic equation, Appl. Math. Lett., 24 (2011), 566-571. 1

[6] R. Barretta, F. Marotti de Sciarra, Constitutive boundary conditions for nonlocal strain gradient elastic nano-beams, Internat. J. Engrg. Sci., 130 (2018), 187-198. 1

[7] A. Bellen, M. Zennaro, Numerical methods for delay differential equations, Oxford University Press, New York, (2003). 1

[8] V. Daftardar-Gejji, Fractional Calculus and Fractional Differential Equations, Birkhäuser, Singapore, (2019). 1

[9] M. M. Elborai, M. I. Youssef, On stochastic solutions of nonlocal random functional integral equations, Arab J. Math. Sci., 25 (2019), 180-188. 2

[10] A. El-Sayed, R. Gamal, Infinite point and Riemann-Stieltjes integral conditions for an integro-differential equation, Nonlinear Anal. Model. Control, 24 (2019), 733-754. 1, 1, 6.1, 6.3, 6.5, 8

[11] M. A. E. Herzallah, Notes on some fractional calculus operators and their properties, J. Fract. Calc. Appl., 5 (2014), 10 pages. 2.1, 3.1, 4.1

[12] U. N. Katugampola, New approach to a generalized fractional integral, Appl. Math. Comput., 218 (2011), 860-865. 2

[13] U. N. Katugampola, A new approach to generalized fractional derivatives, Bull. Math. Anal. Appl., 6 (2014), 1-15. 1

[14] A. I. Kozhanov, Nonlocal problems with integral conditions for elliptic equations, Complex Var. Elliptic Equ., 64 (2019), 741-752. 1

[15] L. T. P. Ngoc, N. A. Triet, A. P. N. Dinh, N. T. Long, Existence and exponential decay of solutions for a wave equation with integral nonlocal boundary conditions of memory type, Numer. Funct. Anal. Optim., 38 (2017), 1173-1207. 1

[16] A. Ricardo, Variational Problems Involving a Caputo-Type Fractional Derivative, J. Optim. Theory Appl., 174 (2017), 276-294. 1, 2 
[17] H. G. Sun, Y. Zhang, D. Baleanu, W. Chen, Y. Q. Chen, A new collection of real world applications of fractional calculus in science and engineering, Commun. Nonlinear Sci. Numer. Simul., 64 (2018), 213-231. 1

[18] K. Szymańska-Debowska, On the existence of solutions for nonlocal boundary value problems, Georgian Math. J., 22 (2015), 273-279. 1

[19] M. I. Youssef, Caputo-Katugampola fractional Volterra functional differential equations with a vanishing lag function, J. Nonlinear Sci. Appl., 13 (2020), 293-302. 2

[20] M. I. Youssef, On the solvability of a general class of a coupled system of stochastic functional integral equations, Arab J. Basic Appl. Sci., 27 (2020), 142-148. 2 